\title{
Characterization of GATA gene family in Vitis vinifera: genome-wide analysis, expression profiles, and involvement in light and phytohormone response
}

\begin{tabular}{|r|l|}
\hline Journal: & Genome \\
\hline Manuscript ID & gen-2018-0042.R2 \\
\hline Manuscript Type: & Article \\
\hline Author: & $30-J$ un-2018 \\
\hline $\begin{array}{r}\text { Complete List of Authors: } \\
\text { zhang, zhan; Institute of Botany Chinese Academy of Sciences; } \\
\text { University of the Chinese Academy of Sciences } \\
\text { ren, chong; Institute of Botany Chinese Academy of Sciences; } \\
\text { University of the Chinese Academy of Sciences } \\
\text { zou, luming; Institute of Botany Chinese Academy of Sciences; } \\
\text { University of the Chinese Academy of Sciences } \\
\text { wang, yi; Institute of Botany Chinese Academy of Sciences; } \\
\text { University of the Chinese Academy of Sciences } \\
\text { li, shaohua; Institute of Botany Chinese Academy of Sciences } \\
\text { liang, zhenchang; Institute of Botany Chinese Academy of } \\
\text { Sciences; Sino-Africa Joint Research Center, Chinese Academy } \\
\text { of Sciences, Wuhan }\end{array}$ \\
\hline $\begin{array}{r}\text { Is the invited manuscript } \\
\text { for consideration in a } \\
\text { Special Issue? : }\end{array}$ & $\begin{array}{l}\text { Grape (Vitis vinifera L.), GATA transcription factor, Evolution, } \\
\text { Expression analysis }\end{array}$ \\
\hline Not applicable (regular submission) \\
\hline
\end{tabular}


1 Characterization of GATA gene family in Vitis vinifera: genome-wide

2 analysis, expression profiles, and involvement in light and

3 phytohormone response

4 Authors: Zhan Zhang ${ }^{1,2}$, Chong Ren ${ }^{1,2}$, Luming Zou ${ }^{1,2}$, Yi Wang ${ }^{1,2}$, Shaohua Li ${ }^{1}$,

5 Zhenchang Liang ${ }^{1,3 *}$

$6 \quad{ }^{1}$ Beijing Key Laboratory of Grape Science and Enology, and CAS Key Laboratory of

7 Plant Resources, Institute of Botany, the Chinese Academy of Science, Beijing, 100093,

8 China

$9 \quad{ }^{2}$ University of Chinese Academy of Sciences, Beijing 100049, China

$10{ }^{3}$ Sino-Africa Joint Research Center, Chinese Academy of Sciences, Wuhan, 430074,

11 China

12 *Corresponding authors:

13 Zhenchang Liang (Email zl249@ibcas.ac.cn) 


\section{Abstract}

24 The plant GATA family is one of the most important transcription factors involved in

25 light-responsive development, nitrogen metabolism, phytohormone signaling, and

26 source/sink balance. However, the function of the GATA gene is less known in grape

27 (Vitis vinifera L.). In this study, we comprehensively analyzed the GATA family in grape, particularly the phylogenetic evolution, duplication patterns, conserved motifs, gene structures, cis-elements, tissue expression patterns, and predicted function of $V v G A T A$ genes in response to abiotic stress. The potential roles of $V v G A T A$ genes in berry development were also investigated. The GATA transcription factors displayed expression diversity among different grape organs and tissues, and some of them showed preferential expression in a specific tissue. Heterotrophic cultured cells were used as model systems for the functional characterization of the $V v G A T A$ gene and study of its response to light and phytohormone. Results indicated that some $V v G A T A$ genes displayed differential responses to light and phytohormones, suggesting their role in light

37 and hormone signaling pathways. A thorough analysis of GATA transcription factors in grape ( $V$. vinifera L.) presented the characterization and functional prediction of $V v G A T A$ genes. The data presented here lay the foundation for further functional studies of grape

40 GATA transcription factors.

41 Keywords Grape (Vitis vinifera L.), GATA transcription factor, Evolution, Expression 42 analysis 


\section{Introduction}

The zinc-finger domain is found in many proteins involved in protein-DNA interactions;

47 GATA transcription factors encode a type IV zinc-finger protein that binds to the DNA sequence $(\mathrm{A} / \mathrm{T}) \mathrm{GATA}(\mathrm{A} / \mathrm{G})$ and acts as regulators of gene expression (Pavletich and Pabo 1991; Lowry and Atchley 2000). GATA transcription factors are encoded by small multigene families, and their protein sequences contain either one or two conserved $\mathrm{CX}_{2} \mathrm{CX}_{17-18} \mathrm{CX}_{2} \mathrm{C}$ motif (Teakle and Gilmartin 1998). Animal GATA factors were originally identified to contain $\mathrm{CX}_{2} \mathrm{CX}_{17} \mathrm{CX}_{2} \mathrm{C}$ domains, and the first GATA gene Eryfl was identified in animals and found to activate all erythroid-specific genes (Evans et al.

54 1988; dEvans and Felsenfeld 1989; Tsai et al. 1989). Subsequent research revealed that

55 GATA factors are critical to the growth and development of vertebrates and invertebrates

56 (Patient and McGhee 2002; Wlodarski et al. 2017; Fujiwara 2017). A fungal GATA

57 factor generally contains a $\mathrm{CX}_{2} \mathrm{CX}_{17} \mathrm{CX}_{2} \mathrm{C}$ or $\mathrm{CX}_{2} \mathrm{CX}_{18} \mathrm{CX}_{2} \mathrm{C}$ domain and is involved in nitrogen metabolism or light response (Teakle and Gilmartin 1998; Wong et al. 2008; Hunter et al. 2014; Niehaus et al. 2017). In plants, GATA factors were originally identified in the regulatory regions of light-responsive genes; some light-inducible genes contain GATA motifs in their promoter regions, and an individual GATA motif that combines with other light-responsive elements and responds to light signals (Puente et al. 
64 al. 2002; Jeong and Shih 2003). The first plant GATA gene (NTL1) was cloned from

65 tobacco (Nicotiana tabacum). A foundation study predicted the existence of 29 and 28

66 GATA genes in Arabidopsis and rice, respectively. Furthermore, most of plant GATA

67 factors contain a $\mathrm{CX}_{2} \mathrm{CX}_{18} \mathrm{CX}_{2} \mathrm{C}$ domain, and several genes contain a $\mathrm{CX}_{2} \mathrm{CX}_{20} \mathrm{CX}_{2} \mathrm{C}$

68 motif or encode a two-zinc finger domain, according to structural analysis results

69 (Daniel-Vedele and Caboche 1993; Reyes et al. 2004).

70 In higher plants, GATA genes are involved in various biological processes, including

71 light response, nitrogen metabolism, chloroplast biogenesis, germination, floral

72 development, and phytohormone signaling. The Arabidopsis GATA2 is a key light-

73 signaling transcription factor that mediates photomorphogenesis (Luo et al. 2010), and

$74 G N C$ and $C G A 1$ are master transcriptional regulators involved in Arabidopsis chloroplast

75 biogenesis (Chiang et al. 2012). B-GATA genes, one of the GATA factor subfamilies

76 from Arabidopsis, have been recently characterized in multiple developmental processes.

77 For instance, the LLM-domain B-GATA genes GNC and CGA1/GNL control

78 germination, flowering, and senescence; some LLM-domain B-GATA genes play

79 important roles in greening, floral development, senescence, cell divisions, and stomata

80 formation (Behringer et al. 2014; Behringer and Schwechheimer 2015; Ranftl et al. 2016;

81 Klermund et al. 2016). The HAN-(HANABATARANU) domain B-GATA gene $(H A N)$ is

82 required in early embryo development of Arabidopsis (Nawy et al. 2010). In monocot

83 rice, $N L 1$ plays an important role in organ differentiation and reproductive development

84 (Wang et al. 2009). OsGATA12 regulates chlorophyll content, senescence, and yield in 
85 rice (Lu et al. 2017). In woody plant species, poplar GATA transcription factor $P d G N C$ regulates chloroplast ultrastructure and photosynthesis (An et al. 2014). Furthermore,

87 GATA factors are responsive to hormone signals, such as auxin and gibberellin signals, which regulate the downstream target genes $G N C$ and $G N L$ during plant growth and development; these signals also regulate brassinosteroid, which participates in the regulation of the GATA transcription factor GATA2 during Arabidopsis photomorphogenesis (Luo et al. 2010; Richter et al. 2013).

93 Reports on the GATA transcription factors in grape are few. To elucidate and explore the

94 functions of the grape GATA transcription factors, we performed a genome-wide analysis

97 duplication events in grape GATA transcription factors. The expression patterns of

103 Methods

\section{Sequence retrieval and identification of $V v G A T A$ genes}


106 performed using the HMMER software (http://www.hmmer.org/). First, we downloaded

107 the Arabidopsis GATA protein sequences from the Arabidopsis Information Resource

108 (http://www.arabidopsis.org/) according to a previously described sequence database of

109 Arabidopsis (Reyes et al. 2004; Manfield et al. 2007). Then, we constructed a Hidden

110 Markov Model to search for GATA sequences and used it to initially obtained $23 \mathrm{~V}$.

111 vinifera GATA protein sequences from the grape genome sequence. All putative GATA

112 protein sequences in the conserved domains were manually analyzed and confirmed with

113 the programs of the Conserved Domain Search Service

114 (http://www.ncbi.nlm.nih.gov/Structure/cdd/wrpsb.cgi). Finally, we identified 19 grape

115 GATA protein sequences, and the sequence data were all downloaded from the Grape

116 Genome Browser (http://www.genoscope.cns.fr/externe/GenomeBrowser/Vitis/).

117 Phylogenetic analysis, chromosomal location, and gene duplication analyses

118 Multiple alignments of protein sequences were performed by Muscle in MEGA5 with

119 default parameters. Then, a phylogenetic tree was constructed on the basis of the

120 alignment results by using the neighbor-joining method with 1000 bootstrap replicates.

121 The parameters used in tree construction were Poisson model, uniform rates, and pairwise

122 deletion.

123 The chromosome map constructed by the Circos software (http://circos.ca/) and

$124 V v G A T A$ gene distribution were determined according to their position information in the

125 Grape Genome Browser (http://www.genoscope.cns.fr/externe/GenomeBrowser/Vitis/).

126 To identify the duplication events of $V v G A T A$ genes, we analyzed 26,346 protein-coding 
127 genes from the grape genomic database, using the MCScanX software

128 (http://chibba.pgml.uga.edu/mcscan2/), The grape protein-coding genes were classified

129 into various types of duplications including tandem duplication and segmental

130 duplication with default parameters (Wang et al. 2012).

\section{Conserved motif, gene structure, and cis-element analyses}

132 The protein sequences of VvGATA were analyzed by Multiple Em for Motif Elicitation

133 (MEME) tools (http://meme-suite.org/tools/meme) for conserved motif (Bailey et al.

134 2006). We set the parameters as follows: motif site distribution; any number of

135 repetitions; maximum number of motifs, 15; and minimum and maximum width of the 136 motif, 6 and 80 respectively. Other parameters had default values.

137 The corresponding genomic DNA sequences and cDNA sequences were obtained

138 from the Grape Genome Browser

139 (http://www.genoscope.cns.fr/externe/GenomeBrowser/Vitis/). Then, the exon and intron

140 structures of $V v G A T A$ genes were analyzed by Gene Structure Display Server

141 (http://gsds.cbi.pku.edu.cn/) (Guo et al. 2007).

142 The $2000 \mathrm{bp}$ promoter sequences of the $19 V v G A T A$ genes were obtained from the

143 Grape Genome Browser (http://www.genoscope.cns.fr/externe/GenomeBrowser/Vitis/).

144 The cis-acting regulatory elements were determined by PLANT CARE

145 (http://bioinformatics.psb.ugent.be/webtools/plantcare/html/) with default parameters.

$146 \quad$ Plant materials and treatments

147 Grape seedlings ( $V$. vinifera L. cv. Cabernet Sauvignon) and suspension-cultured cells 
148 were used. Cultivar Cabernet Sauvignon is one of the most widely cultivated and

149 economically important fruit crop in the world, and the use of suspension-cultured cells

150 in the study of exogenous treatment offers an advantage in bulk diffusion. Cultivar

151 Cabernet Sauvignon was grown in the grape germplasm resource garden of the Institute

152 of Botany, the Chinese Academy of Sciences, Beijing, under natural conditions. Roots,

153 stems, adult leaves, flowers, and berries at different development stages (fruit setting [F],

154 veraison [V], and ripening [R]) were sampled for gene expression analysis.

155 Cabernet Sauvignon berry suspension cells were cultured in $250 \mathrm{~mL}$ flasks filled

156 with $100 \mathrm{~mL}$ of liquid B5 medium (B5 basal medium supplemented with $2.5 \mathrm{~g} / \mathrm{L}$

157 hydrolyzed casein, $0.2 \mathrm{mg} / \mathrm{L} \mathrm{KT}, 0.1 \mathrm{mg} / \mathrm{L} \mathrm{NAA}$, and $30 \mathrm{~g} / \mathrm{L}$ sucrose) and centrifuged at

$158120 \mathrm{rpm}$ in light conditions at $25 \pm 1{ }^{\circ} \mathrm{C}$ (Zhang et al. 2014). All the suspension cells

159 were subcultured every 7 days. Cabernet Sauvignon berry callus was also cultured in

160 solid B5 medium (liquid B5 medium supplemented with $3 \mathrm{~g} / \mathrm{L}$ Phytagel) under light

161 conditions at $25 \pm 1{ }^{\circ} \mathrm{C}$. The berry callus was subcultured every month. The culture

162 protocol was based on the method described previously with some modifications (Zhang

163 et al. 2014).

164 Berry callus was grown on solid B5 medium for 2 weeks (logarithmic growth phase

165 of cells), then the callus was subcultured for the light and dark treatments. For the light

166 exclusion treatment, the culture flasks were wrapped in aluminum foil, and the flasks

167 were covered with a shade cloth and placed at $25 \pm 1^{\circ} \mathrm{C}$ for 2 weeks. For the light-grown

168 treatment, the subculture callus was cultured with light/dark $(16: 8 \mathrm{~h})$ conditions at $25 \pm$ 
$1691^{\circ} \mathrm{C}$. The calluses of the light/dark treatment groups were harvested after two weeks, 170 frozen in liquid nitrogen, and stored at $-80{ }^{\circ} \mathrm{C}$ for further analysis. For the plant hormone 171 treatment, the suspension-cultured cells were harvested at logarithmic phase (5-6 days 172 after subculture), washed three times with sterile deionized water, and transferred into a $173100 \mathrm{~mL}$ of hormone-free liquid B5 medium. Then, indole-3-acetic acid (IAA), gibberellin 174 A3 (GA3), abscisic acid (ABA), and 2,4-epicastasterone (EBR) were added into the 175 hormone-free liquid B5 medium to a final concentration of $50 \mu \mathrm{M}$. After the hormones 176 were added, the suspension-cultured cells were harvested at 0,6 , and $12 \mathrm{~h}$. The 177 suspension-cultured cells were centrifuged at $5000 \mathrm{rpm}$ for $5 \mathrm{~min}$ for the collection of the 178 cells. The collected cells were frozen in liquid nitrogen and stored at $-80{ }^{\circ} \mathrm{C}$ for further 179 analysis. All the treatments were replicated two or three times.

\section{RNA extraction and real-time PCR analysis}

181 Total RNA was extracted from the different tissues and organs, berry callus, and 182 suspension-cultured cells of Cabernet Sauvignon. An RNAprep pure plant kit (Qiagen, 183 China) was used for the extraction according to the manufacturer's instructions. For the 184 first-strand cDNA synthesis, the RNA quality was measured by agarose gel 185 electrophoresis, and concentration was detected by a NanoDrop 2000 spectrophotometer 186 (Thermo Scientific, USA). First-strand cDNA was synthesized from 500 ng of total RNA 187 with a HiScript Q RT SuperMix for qPCR (+ gDNA wiper) Kit (Vazyme, China). RNA 188 extraction and reverse transcription steps conform to those outlined in the Minimum 189 Information for Publication of Quantitative Real-Time PCR Experiments guidelines 
190 (Bustin, et al. 2009). The qPCR results were completed with the CFX96 System (Bio-

191 Rad, USA), and an AceQ qPCR SYBR Green Master Mix (Vazyme, China) was used.

192 The reaction conditions were as follows: $95^{\circ} \mathrm{C}$ for $5 \mathrm{~min}$, followed by 40 cycles of $95{ }^{\circ} \mathrm{C}$

193 for $10 \mathrm{~s}$ and $60{ }^{\circ} \mathrm{C}$ for $30 \mathrm{~s}$. The primer sequences are listed in Table S1. Grape Actin1

194 (accession no. AY680701) was used as an internal standard, and the relative expression

195 change was calculated by the formula $2^{-\Delta \Delta \mathrm{Ct}}$ (Livak and Schmittgen 2001).

196 Protoplast isolation and transfection

197 The whole coding sequence of $V v G A T A$ was amplified from cDNA from Cabernet

198 Sauvignon berry with gene specific primers (Table S1). The PCR fragment was digested

199 with EcoR I and Kpn I, then it was subcloned into the pEZS-NL vector for the generation

200 of $p E Z S-N L-V v G A T A-G F P$, which was driven by the $35 \mathrm{~S}$ CaMV promoter and contains a

$201 V v G A T A-G F P$ fusion construct. The construct was confirmed by sequencing. Given that

202 the largescale isolation of viable grape protoplasts is difficult, the tobacco (Nicotiana

203 benthamiana) leaf of seed tissues cultured on the MS medium was used for protoplast

204 isolation, and the method used for this step was performed according to the protocol of $A$.

205 thaliana and grapevine mesophyll protoplast isolation (Yoo et al. 2007; Zhao et al. 2016).

206 Protoplasts were transfected through a polyethylene glycol method described previously

207 (Zhao et al. 2016). Transfected protoplasts were incubated for $12-16 \mathrm{~h}$ at $25^{\circ} \mathrm{C}$ in the

208 dark. The fluorescence signals were analyzed by confocal microscopy (Leica TCS SP5,

209 Germany). GFP fluorescent signals were excited at $488 \mathrm{~nm}$, and auto-fluorescence was

210 captured at 505-535 $\mathrm{nm}$. The transient expression experiment was repeated at least three 
211 times.

\section{Results}

\section{Identification of the GATA factor family in grape}

214 For the identification of potential GATA genes in grape, a BLAST program was carried

215 out by using the 29 Arabidopsis GATA proteins against the grape genome sequence

216 (Reyes et al. 2004; Manfield et al. 2007). We constructed a hidden Markov model to

217 search for the GATA sequences. All these putative sequences were analyzed in the

218 conserved GATA zinc finger domain at the NCBI Conserved Domain Search Service. A

219 total of 19 GATA genes were identified. This result was consistent with that obtained in

220 the Plant Transcription Factor Database (PlantTFDB, http://planttfdb.cbi.pku.edu.cn/). A

221 summary of the gene ID, conserved domain accession number, and short name of the 19

222 GATA genes are listed in Table S2.

223 We named these 19 GATA genes according to phylogenetic analysis results and

224 those that best matched the Arabidopsis GATA proteins (Table 1 and Fig. 1). VvGATA-

225 encoded peptides ranged from 109 to 386 amino acids and were classified into four

226 subfamilies (Table 1). These GATA genes were distributed on 11 grape chromosomes,

227 which displayed an unbalanced distribution. Four $V v G A T A$ ( $V v G A T A 2, V v G A T A 5$,

$228 V v G A T A 9, V v G A T A 10)$ genes were mapped on chromosome 4; three $V v G A T A$

229 ( $V v G A T A 3, V v G A T A 16$, and $V v G A T A 18)$ genes on chromosome 3; two on chromosomes

2309 9, 14, and 18; and only one gene on chromosomes 5, 6, 8, 11, 12 and 15 (Fig. 2).

$231 V v G A T A 15$ and $V v G A T A 17$ were separated by only $1.8 \mathrm{~kb}$ on chromosome 18. 
232 Phylogenetic relationships, conserved motifs, and gene structures of GATA genes in 233 grape

234 To investigate the phylogenetic relationships of GATA genes, we constructed a 235 phylogenetic tree, using the GATA protein sequences from grape and Arabidopsis (Fig. 236 1). The result revealed that $19 V v G A T A$ genes were divided into four subgroups (I, II, III, 237 and IV), which had 7, 6, 5, and $1 V v G A T A$ members, respectively. The grape GATA 238 family is contracted in contrast to that of Arabidopsis. Three subgroups (I, II and IV) are 239 contracted in grape. Each subgroup had one or more pairs of GATA proteins with a high 240 bootstrap value. This finding suggested that the $V v G A T A$ genes in each subgroup have a 241 common origin. Moreover, the GATA genes from the same lineage tended to cluster 242 together in the phylogenetic tree. This suggested that they experienced duplications after 243 the divergence of grape and Arabidopsis. And no species-specific subgroups were 244 observed, implying that GATA genes were more conserved during evolution. The 245 functions of some AtGATA genes are well characterized in Arabidopsis; therefore, the 246 evolutionary relationship of GATA genes between grape and Arabidopsis may provide 247 insights into their roles in grape development. Gene duplication events play an important role in the evolution of a gene family, and tandem and segmental duplications are the two main mechanisms (Cannon et al. 2004). To detect the potential evolutionary relationships among $V v G A T A$ genes, we analyzed tandem duplication and segmental duplication events within the grape genome.

252 The result indicated that $17 V v G A T A$ genes underwent segmental duplications and one 
253 pair of genes ( $V v G A T A 15$ and $V v G A T A 17)$ was assigned to a tandem duplication (Fig. 2),

254 suggesting that segmental duplication events have played a major role in the expansion of 255 the $V v G A T A$ gene family.

256 Conserved motifs and gene structures play important roles in multiple gene families, 257 and revealing the diversity of motifs and gene structures may provide an explanation for 258 the evolution of gene families. To reveal the diversification of $V v G A T A$ genes in grape, 259 we analyzed the conserved motifs. The analysis was performed on a MEME program. 260 Ten distinct conserved motifs were identified in 19 VvGATA proteins (Fig. 3a). All the 261 grape GATA genes contained a GATA zinc finger. VvGATA14, VvGATA15, (pfam06203) apart from a GATA zinc-finger domain (Table S2). boundaries. The results showed that genes in the same subgroup generally had the same exon/intron number, position, or length (Fig. 3b). For example, $V v G A T A 14, V v G A T A 15$, $V v G A T A 16$, and $V v G A T A 17$ showed similar gene structure patterns. Different subgroups had different in gene structure. For instance, most members of subfamily I contain three exons. The genes of subgroup III contain seven, eight or eleven exons, and these genes had long introns. The variation in the structures of grape GATA gene family indicates that the $V v G A T A$ genes changed during their evolutionary history. This change might have led

272 to the considerable functional diversity in the $V v G A T A$ gene family. 
274 To verify the cis-elements involved in the regulation of $V v G A T A$ genes, we obtained

$2752000 \mathrm{bp}$ DNA sequences upstream of the $V v G A T A$ genes and identified cis-acting

276 regulatory elements. PLANT CARE

277 (http://bioinformatics.psb.ugent.be/webtools/plantcare/html/) was used for the

278 identification. We identified 99 cis-elements, and most of them were classified as light-

279 responsive elements (Table S3). Some cis-elements were highly conserved in the

280 promoter region of the $V v G A T A$ genes, and 17 cis-acting regulatory elements were

281 ubiquitous among the $V v G A T A$ gene members (Table 2). The cis-elements were

282 responsive to light, circadian rhythm, plant hormones, and abiotic or biotic stresses. For

283 instance, two light-responsive elements (Box 4, Box I), a heat-stress element (HSE), and

284 an endosperm expression element (Skn-1_motif) were represented in nearly all the

$285 V v G A T A$ genes. Plant hormones, such as abscisic acid, salicylic acid, gibberellin, MeJA,

286 ethylene, and auxin, also had a responsive motif in the promoter region of the $V v G A T A$

287 members (Table 2, Table S3).

288 Expression profiles of $V \boldsymbol{v} G A T A$ genes in different grape tissues

289 We analyzed the expression pattern of the $V v G A T A$ genes in the roots, stems, adult

290 leaves, flowers, and berries of grape ( $V$. vinifera L. cv. Cabernet Sauvignon) at fruit

291 setting [F], veraison [V], and ripening [R] (Fig. 4). Quantitative RT-PCR results showed

292 that all $V v G A T A$ genes were expressed, except $V v G A T A 4$ and $V v G A T A 19$, and most of

293 the $V v G A T A$ genes displayed higher expression levels in vegetative organs than in berry

294 tissues (Fig. 4). Some $V v G A T A$ genes showed minimal variation among organs and 
295

296

297

298

299

300

301

302

tissues (Fig. 4a), and other $V v G A T A$ genes displayed wider variation (Fig. 4b). For instance, $V v G A T A 1, V v G A T A 3, V v G A T A 6, V v G A T A 10$, and $V v G A T A 11$, were highly expressed in the leaves, $V v G A T A 5$ and $V v G A T A 7$ in the flowers, and $V v G A T A 3$ in the roots. Notably, VvGATA11 displayed the highest expression level in the adult leaves.

Overall, most of $V v G A T A$ genes are highly expressed in vegetative organs. The expression patterns in vegetative organs indicate that $V v G A T A$ genes have important roles in source tissues.

RNA-seq data are useful resources for studying gene transcript abundance. To obtain insight into the transcript patterns of the $V v G A T A$ genes during fruit development, we analyzed the three developmental stages of berry (fruit setting [F], veraison [V], and ripening [R]) of Cabernet Sauvignon RNA-seq data from our unpublished database (Fig.

4c). The $V v G A T A$ genes were divided into four main clades, and the transcript abundances of $V v G A T A 9, V v G A T A 11$, and $V v G A T A 19$ were not detected in the berries.

Clade 1 contained four genes. $V v G A T A 17$ was highly expressed in all stages, whereas the other genes displayed preferential expression in some stages. VvGATA16 in clade 2 exhibited the highest stable expression in the berry tissues. The genes in clade 3 exhibited high expression levels in the fruit setting stage. By contrast, the genes in clade 4 were lowly expressed during berry development.

\section{Subcellular localization of the VvGATA proteins}

Generally, grape GATA proteins contain a conserved GATA zinc-finger domain. However, in addition to VvGATA18, subgroup III GATA proteins also contain two other 
316 conserved domains (CCT, TIFY). To determine VvGATA subcellular localization, we

317 selected VvGATA14, VvGATA15 and VvGATA18 as reference proteins for nuclear

318 targeting. The entire coding sequence of $V v G A T A$ from $V$. vinifera L. cv. Cabernet

319 Sauvignon was fused in frame to the GFP coding sequence under the control of the

320 CaMV 35S promoter, and the recombinant constructs were transfected into tobacco

321 protoplasts. As shown in Fig. 5, the pEZS-NL-VvGATA14-GFP and pEZS-NL-

322 VvGATA15-GFP fusion proteins predominantly accumulated in the nucleus, whereas the

323 pEZS-NL-VvGATA18-GFP was targeted to the nucleus, although the accumulation of

324 the fusion protein was not restricted to the nucleus. The VvGATA18 protein was also

325 located at the extranuclear compartment. These results indicate that nuclear targeting of

326 the VvGATA protein is consistent with their predicted transcriptional regulatory

327 function.

328 Expression patterns of $V \boldsymbol{v} G A T A$ genes in response to light and phytohormones

329 We investigated $V v G A T A$ gene functions by using heterotrophic-cultured cells obtained

330 from grape berries as a model system. We focused on grape GATA transcription factors

331 that play important roles in berry development. Our quantitative RT-PCR results and

332 RNA-seq data revealed that $V v G A T A$ genes in subgroup III ( $V v G A T A 14, V v G A T A 15$,

$333 V v G A T A 16, V v G A T A 17, V v G A T A 18)$ were more highly expressed than those in the other

334 subgroups in berries. Thus, we assayed the expression levels of grape GATA subgroup

335 III genes under light/dark treatment. As shown in Fig. 6a, the selected $V v G A T A$ genes

336 (subgroup III) were all up-regulated by light, and the transcript abundance of $V v G A T A 18$ 
337 showed twofold increase under light treatment. VvGATA14 and VvGATA16 showed 1.5-

338 fold difference between the light-grown and dark-grown berry callus, whereas the others

339 showed only a slight difference. These results indicated that the $V v G A T A$ genes are

340 involved in light-responsive regulation.

341 Phytohormones play an important role in the berry development of grape. To

342 determine whether $V v G A T A$ genes are responsive to hormone signaling, we analyzed the

343 expression level of the selected $V v G A T A$ genes (subgroup III) by using exogenous indole-

344 3-acetic acid (IAA), gibberellin A3 (GA3), abscisic acid (ABA), and 2,4-epicastasterone

345 (EBR) treatments. The expression of $V v G A T A 14$ and $V v G A T A 16$ displayed continuous

346 up-regulation for $12 \mathrm{~h}$, whereas the other genes showed slight variation under exogenous

347 IAA treatment (Fig. 6b). VvGATA14 transcript accumulation increased after the GA3 and

348 ABA treatments (Fig. 6c, d), whereas the transcript accumulation of $V v G A T A 17$ or

$349 V v G A T A 18$ was down-regulated under the GA3 treatment (Fig. 6c). Additionally, all the

350 selected $V v G A T A$ genes showed up-regulation after EBR treatment (Fig. 6e).

351 Interestingly, $V v G A T A 14$ displayed response to all these phytohormones. These data

352 suggest that $V v G A T A$ genes might also be involved in phytohormone signaling pathway.

353 Discussion

354 GATA transcription factors are important to plant development. In this study, we showed

355 a fundamental exploration and analysis of the grape GATA gene family, and provided

356 insight into the potential functions of the $V v G A T A$ genes in light and phytohormone

357 response during grape berry development. To identify the grape GATA transcription 
358 factors, we performed a genome-wide scan of the grapevine genome sequence (Jaillon et

al. 2007). A total of $19 V v G A T A$ protein-encoding genes were identified. Meanwhile, a phylogenetic tree was constructed to predict the evolutionary relationships of $V v G A T A$ genes and the potential functions based on homologous genes (AtGATA2, GNC, GNL, and $H A N$ ) that have been well studied in Arabidopsis. The grape GATA family was classified into four major subfamilies (I, II, III, and IV; Fig. 1). However, subgroup III was substantially expanded in grape. Arabidopsis subgroup III genes were found to contain a $\mathrm{CX}_{2} \mathrm{CX}_{20} \mathrm{CX}_{2} \mathrm{C}$ motif, exclusively conserved domain CCT, and TIFY (ZIM), which belong to a novel plant-specific GATA-type transcription factors (Shikata et al. 2004; Vanholme et al. 2007). VvGATA proteins in subgroup III also contain this domain (VvGATA14, VvGATA15, VvGATA16, and VvGATA17). ZIM proteins or TIFY proteins are involved in petiole and hypocotyl elongation, flower development, or root development in Arabidopsis (Nishii et al. 2000; Shikata et al. 2004; Vanholme et al. 2007), and our expression pattern analysis showed that $V v G A T A$ genes in subgroup III were more highly expressed during berry developmental stages than the other $V v G A T A$ genes. This result indicated that $V v G A T A$ genes in subgroup III might be involved in sink organ developmental processes. B-GATAs, also known as Arabidopsis subgroup II genes were identified to play a key role in plant growth and development, such as in greening and flowering (Behringer and Schwechheimer 2015). Considering these B-GATA genes, $G N C, C G A 1 / G N L$, and $H A N$, were well-characterized, the grape subgroup II homologous genes might be candidate genes for further study. 

subgroup I, and both of them showed high expression level in the leaves. Furthermore,

VvGATA6. These results implied the potential roles of these genes in light-regulated 
400 Therefore, further study of the functional difference among plant species is necessary. In

401 grape subgroup II, $V v G A T A 8$ was highly expressed in grape organs, especially in the

402 stem, leaf, and flower. The orthologous genes of VvGATA8 in Arabidopsis are AtGATA15

403 and AtGATA16, which participate in greening, hypocotyl elongation, phyllotaxy, floral

404 organ initiation, accessory meristem formation, flowering time, and senescence (Ranftl et

405 al. 2016). Likewise, the orthologous gene in rice, OsGATA12, regulates chlorophyll

406 levels, senescence, and yield parameters (Lu et al. 2017). These results suggest that

$407 V v G A T A 8$ plays an important role in grape growth and development. Thus, $V v G A T A 8$

408 should be considered a candidate gene for developmental responses in grape. The GATA

409 factor HAN (AtGATA18) mediates embryogenesis and flower development in

410 Arabidopsis (Zhao et al. 2004; Nawy et al. 2010; Zhang et al. 2013). However, the

411 homologous gene $V v G A T A 9$ displayed low expression levels in different grape organs

412 and tissues. VvGATA10 and VvGATA11 displayed tissue-specific expression; in

413 particular, $V v G A T A 11$ displayed high-level of expression in leaves. $G N C$, a homologous

414 gene of $V v G A T A 10$ and $V v G A T A 11$, exhibits multiple biological functions in higher

415 plants, particularly in germination, greening, chlorophyll synthesis, flowering time,

416 senescence, and glucose sensitivity (Bi et al. 2005; Chiang et al. 2012; Behringer et al.

417 2014; Behringer and Schwechheimer 2015; Ranftl et al. 2016; Klermund et al. 2016).

418 These results provide an insight into the potential function of VvGATA10 and VvGATA11

419 in regulating carbon metabolism and other biological processes.

420 In grape berry tissues, some $V v G A T A$ genes in subgroup III displayed higher 
421

422

423

424

425

426

427

428

429

430

431

432

433

434

435

436

437

438

439

440

441

expression than the other $V v G A T A$ genes according to our RNA-seq data (Fig. 4c).

VvGATA14 displayed higher expression before veraison, $V v G A T A 15$ was mainly

expressed in fruit setting and ripening stages, and $V v G A T A 16$ and $V v G A T A 17$ were

highly expressed in all berry developmental stages. Thus, $V v G A T A 14, V v G A T A 15$,

VvGATA16, and $V v G A T A 17$ should be candidate genes for further study in berry

development. The subcellular localization of VvGATA14, VvGATA15, and VvGATA18

displayed the nuclear localization of VvGATA, and this result is consistent with the

regulatory function of VvGATA as a transcription factor. Many studies demonstrated that

light or phytohormones play important roles in grape growth and berry development

(Deluc et al. 2007; Kuhn et al. 2013; Böttcher et al. 2013; Kondo et al. 2014; Xu et al.

2015) and some GATA genes are responsive to light or hormone signals (Luo et al. 2010;

Richter et al. 2013). In this study, $V v G A T A 18$ and $V v G A T A 14$ were significantly up-

regulated under light treatment and exogenous phytohormone treatment, respectively.

In this study, we provided basic information about $V v G A T A$ genes. Nevertheless, the details of the molecular regulation mechanism of the VvGATA transcription factors in grape still needs further study.

Author contribution statement ZCL, SHL and ZZ designed the experiments. ZZ performed the experiments, analyzed results and manuscript preparation. CR, LMZ and YW performed bioinformatics analyses. All authors read and approved the final manuscript.

Acknowledgements This work was supported by the National Natural Science 
442 Foundation of China (NO. 31772266 and 31572090), Agricultural Breeding Project of

443 Ningxia Hui Autonomous Region (NXNYYZ20150203) and Hundred Talents of Chinese

444 Academy of Sciences.

445 Compliance with ethical standards

446 Conflict of interest

447 The authors declare that they have no conflict of interest.

448

449

450

451

452

453

454

455

456

457

458

459

460

461

462 


\section{References}

467 An, Y., Han, X., Tang, S., Xia, X., and Yin, W. 2014. Poplar GATA transcription factor

468 PdGNC is capable of regulating chloroplast ultrastructure, photosynthesis, and vegetative

469 growth in Arabidopsis under varying nitrogen levels. Plant Cell, Tissue Organ Cult

470

119(2): 313-327. doi: $\underline{10.1007 / \mathrm{s} 11240-014-0536-\mathrm{y}}$

471 Argüello-Astorga, G., and Herrera-Estrella, L. 1998. Evolution of light-regulated plant

472 promoters. Annu. Rev. Plant Biol. 49(1): 525-555.

473 Available from https://doi.org/10.1146/annurev.arplant.49.1.525

474 Böttcher, C., Burbidge, C.A., Boss, P.K., and Davies, C. 2013. Interactions between

475 ethylene and auxin are crucial to the control of grape (Vitis vinifera L.) berry ripening.

476 BMC Plant Biol. 13(1): 222. Available from https://doi.org/10.1186/1471-2229-13-222

477 Bailey, T.L., Williams, N., Misleh, C., and Li, W.W. 2006. MEME: discovering and

478 analyzing DNA and protein sequence motifs. Nucleic Acids Res. 34: W369-W373.

479 Available from https://doi.org/10.1093/nar/gk1198

480 Behringer, C., Bastakis, E., Ranftl, Q.L., Mayer, K.F., and Schwechheimer, C. 2014.

481 Functional diversification within the family of B-GATA transcription factors through the

482 leucine-leucine-methionine domain. Plant Physiol. 166(1): 293-305.

483 Available from https://doi.org/10.1104/pp.114.246660 
484 Behringer, C., and Schwechheimer, C. 2015. B-GATA transcription factors-insights into 485 their structure, regulation, and role in plant development. Front Plant Sci 6:90 Available 486 from https://doi.org/10.3389/fpls.2015.00090

487 Bi, Y.M., Zhang, Y., Signorelli, T., Zhao, R., Zhu, T., and Rothstein, S. 2005. Genetic 488 analysis of Arabidopsis GATA transcription factor gene family reveals a nitrate 489 inducible member important for chlorophyll synthesis and glucose sensitivity. Plant $\mathbf{J}$ 490 44(4): 680-692. Available from https://doi.org/10.1111/j.1365-313X.2005.02568.x

491 Bustin, S.A., Benes, V., Garson, J.A., Hellemans, J., Huggett, J., Kubista, M., Mueller, 492 R., Nolan, T., Pfaffl, M.W., and Shipley, G.L. 2009. The MIQE guidelines: minimum 493 information for publication of quantitative real-time PCR experiments. Clin Chem. 55(4): $494 \quad 611-622$.

495 Available from http://www.clinchem.org/cgi/doi/10.1373/clinchem.2008.112797

496 Cannon, S.B., Mitra, A., Baumgarten, A., Young, N.D., May, G. 2004. The roles of

497 segmental and tandem gene duplication in the evolution of large gene families in 498 Arabidopsis thaliana. BMC Plant Biol. 4:10.

499 Available from https://doi.org/10.1186/1471-2229-4-10

500 Chattopadhyay, S., Puente, P., Deng, X.W., and Wei, N. 1998. Combinatorial interaction 501 of light - responsive elements plays a critical role in determining the response 502 characteristics of light - regulated promoters in Arabidopsis. Plant J 15(1): 69-77.

503 Available from https://doi.org/10.1046/j.1365-313X.1998.00180.x

504 Chiang, Y.-H., Zubo, Y.O., Tapken, W., Kim, H.J., Lavanway, A.M., Howard, L., Pilon, 
505 M., Kieber, J.J., and Schaller, G.E. 2012. Functional characterization of the GATA

506 transcription factors GNC and CGA1 reveals their key role in chloroplast development,

507 growth, and division in Arabidopsis. Plant Physiol. 160(1): 332-348. Available from

508 https://doi.org/10.1104/pp.112.198705

509 Daniel-Vedele, F., and Caboche, M. 1993. A tobacco cDNA clone encoding a GATA-1

510 zinc finger protein homologous to regulators of nitrogen metabolism in fungi. Mol Gen

511 Genet 240(3): 365-373.

512 Available from https://link.springer.com/article/10.1007/BF00280388

513 Deluc, L.G., Grimplet, J., Wheatley, M.D., Tillett, R.L., Quilici, D.R., Osborne, C.,

514 Schooley, D.A., Schlauch, K.A., Cushman, J.C., and Cramer, G.R. 2007. Transcriptomic

515 and metabolite analyses of Cabernet Sauvignon grape berry development. BMC

516 Genomics 8(1): 429. Available from https://doi.org/10.1186/1471-2164-8-429

517 dEvans, T., and Felsenfeld, G. 1989. The erythroid-specific transcription factor Eryf1: a

518 new finger protein. Cell 58(5): 877-885.

519 Available from $\underline{\text { https://doi.org/10.1016/0092-8674(89)90940-9 }}$

520 Evans, T., Reitman, M., and Felsenfeld, G. 1988. An erythrocyte-specific DNA-binding

521 factor recognizes a regulatory sequence common to all chicken globin genes. Proc Natl

522 Acad Sci USA 85(16): 5976-5980.

523 Available from http://www.pnas.org/content/85/16/5976/tab-article-info

524 Fujiwara, T. 2017. GATA Transcription Factors: Basic Principles and Related Human

525 Disorders. Tohoku J Exp Med 242(2): 83-91. doi: 10.1620/tjem.242.83 
526 Guo, A.-Y., Zhu, Q., Chen, X., and Luo, J. 2007. GSDS: a gene structure display server.

527 Yi chuan= Hereditas 29(8): 1023-1026. doi: $\underline{10.1360 / y c-007-1023}$

528 Hunter, C.C., Siebert, K.S., Downes, D.J., Wong, K.H., Kreutzberger, S.D., Fraser, J.A.,

529 Clarke, D.F., Hynes, M.J., Davis, M.A., and Todd, R.B. 2014. Multiple nuclear

530 localization signals mediate nuclear localization of the GATA transcription factor AreA.

531 Eukaryot. Cell 13(4): 527-538. doi: $\underline{10.1128 / \text { EC.00040-14 }}$

532 Jaillon, O., Aury, J.-M., Noel, B., Policriti, A., Clepet, C., Casagrande, A., Choisne, N.,

533 Aubourg, S., Vitulo, N., and Jubin, C. 2007. The grapevine genome sequence suggests

534 ancestral hexaploidization in major angiosperm phyla. Nature 449(7161): 463. Available

535 from https://www.nature.com/articles/nature06148

536 Jeong, M.-J., and Shih, M.-C. 2003. Interaction of a GATA factor with cis-acting

537 elements involved in light regulation of nuclear genes encoding chloroplast

538 glyceraldehyde-3-phosphate dehydrogenase in Arabidopsis. Biochem. Biophys. Res.

539 Commun. 300(2): 555-562.doi: 10.1016/S0006-291X(02)02892-9

540 Klermund, C., Ranftl, Q.L., Diener, J., Bastakis, E., Richter, R., and Schwechheimer, C.

541 2016. Llm-domain b-gata transcription factors promote stomatal development

542 downstream of light signaling pathways in Arabidopsis thaliana hypocotyls. Plant Cell

543 28(3): 646-660. doi: $\underline{10.1105 / \text { tpc. } 15.00783}$

544 Kondo, S., Tomiyama, H., Rodyoung, A., Okawa, K., Ohara, H., Sugaya, S., Terahara,

545 N., and Hirai, N. 2014. Abscisic acid metabolism and anthocyanin synthesis in grape skin

546 are affected by light emitting diode (LED) irradiation at night. J. Plant Physiol. 171(10): 
547 823-829. Available from https://doi.org/10.1016/j.jplph.2014.01.001

548 Kuhn, N., Guan, L., Dai, Z.W., Wu, B.-H., Lauvergeat, V., Gomès, E., Li, S.-H., Godoy,

549 F., Arce-Johnson, P., and Delrot, S. 2013. Berry ripening: recently heard through the

550 grapevine. J. Exp. Bot. 65(16): 4543-4559.

551 Available from https://doi.org/10.1093/jxb/ert395

552 Livak, K.J., and Schmittgen, T.D. 2001. Analysis of relative gene expression data using

553 real-time quantitative PCR and the 2- $\Delta \Delta \mathrm{CT}$ method. Methods 25(4): 402-408. Available

554 from https://doi.org/10.1006/meth.2001.1262

555 Lowry, J.A., and Atchley, W.R. 2000. Molecular evolution of the GATA family of

556 transcription factors: conservation within the DNA-binding domain. J. Mol. Evol. 50(2):

557 103-115. doi: $10.1007 / \mathrm{s} 002399910012$

558 Lu, G., Casaretto, J.A., Ying, S., Mahmood, K., Liu, F., Bi, Y.-M., and Rothstein, S.J.

559 2017. Overexpression of OsGATA12 regulates chlorophyll content, delays plant

560 senescence and improves rice yield under high density planting. Plant Mol. Biol. 94(1-2):

561 215-227. doi: $\underline{10.1007 / \mathrm{s} 11103-017-0604-\mathrm{X}}$

562 Luo, X.-M., Lin, W.-H., Zhu, S., Zhu, J.-Y., Sun, Y., Fan, X.-Y., Cheng, M., Hao, Y.,

563 Oh, E., and Tian, M. 2010. Integration of light-and brassinosteroid-signaling pathways by

564 a GATA transcription factor in Arabidopsis. Dev. Cell 19(6): 872-883. Available from

$565 \quad$ https://doi.org/10.1016/j.devcel.2010.10.023

566 Manfield, I.W., Devlin, P.F., Jen, C.-H., Westhead, D.R., and Gilmartin, P.M. 2007.

567 Conservation, convergence, and divergence of light-responsive, circadian-regulated, and 
568 tissue-specific expression patterns during evolution of the Arabidopsis GATA gene

569 family. Plant Physiol. 143(2): 941-958.

570 Available from https://doi.org/10.1104/pp.106.090761

571 Nawy, T., Bayer, M., Mravec, J., Friml, J., Birnbaum, K.D., and Lukowitz, W. 2010. The

572 GATA factor HANABA TARANU is required to position the proembryo boundary in the

573 early Arabidopsis embryo. Dev. Cell 19(1): 103-113.

574 Available from https://doi.org/10.1016/j.devcel.2010.06.004

575 Niehaus, E.-M., Schumacher, J., Burkhardt, I., Rabe, P., Spitzer, E., Münsterkötter, M.,

576 Güldener, U., Sieber, C.M., Dickschat, J.S., and Tudzynski, B. 2017. The GATA-type

577 transcription factor Csm1 regulates conidiation and secondary metabolism in Fusarium

578 fujikuroi. Front Microbiol 8:1175.

579 Available from https://doi.org/10.3389/fmicb.2017.01175

580 Nishii, A., Takemura, M., Fujita, H., Shikata, M., Yokota, A., and Kohchi, T. 2000.

581 Characterization of a novel gene encoding a putative single zinc-finger protein, ZIM,

582 expressed during the reproductive phase in Arabidopsis thaliana. Biosci., Biotechnol.,

583 Biochem. 64(7): 1402-1409. Available from https://doi.org/10.1271/bbb.64.1402

584 Patient, R.K., and McGhee, J.D. 2002. The GATA family (vertebrates and invertebrates).

585 Curr. Opin. Genet. Dev. 12(4): 416-422. Available from https://doi.org/10.1016/S0959-

$586 \quad \underline{437 X(02) 00319-2}$

587 Pavletich, N.P., and Pabo, C.O. 1991. Zinc finger-DNA recognition: crystal structure of a

588 Zif268-DNA complex at 2.1 A. Science 252(5007): 809. 
589

590

591

592

593

594

595

596

597

598

599

600

601

602

603

604

605

606

607

608

609

doi: $10.1126 /$ science. 2028256

Puente, P., Wei, N., and Deng, X.W. 1996. Combinatorial interplay of promoter elements constitutes the minimal determinants for light and developmental control of gene

expression in Arabidopsis. Embo J 15(14): 3732.

Available from https://doi.org/10.1002/j.1460-2075.1996.tb00743.x

Ranftl, Q.L., Bastakis, E., Klermund, C., and Schwechheimer, C. 2016. LLM-domain

containing B-GATA factors control different aspects of cytokinin-regulated development

in Arabidopsis thaliana. Plant Physiol. 170:2295-2311.

Available from https://doi.org/10.1104/pp.15.01556

Reyes, J.C., Muro-Pastor, M.I., and Florencio, F.J. 2004. The GATA family of

transcription factors in Arabidopsis and rice. Plant Physiol. 134(4): 1718-1732. Available

from https://doi.org/10.1104/pp.103.037788

Richter, R., Behringer, C., Zourelidou, M., and Schwechheimer, C. 2013. Convergence of auxin and gibberellin signaling on the regulation of the GATA transcription factors GNC

and GNL in Arabidopsis thaliana. Proc Natl Acad Sci USA 110(32): 13192-13197.

Available from https://doi.org/10.1073/pnas.1304250110

Shikata, M., Matsuda, Y., Ando, K., Nishii, A., Takemura, M., Yokota, A., and Kohchi,

T. 2004. Characterization of Arabidopsis ZIM, a member of a novel plant - specific

GATA factor gene family. J. Exp. Bot. 55(397): 631-639. Available from

https://doi.org/10.1093/jxb/erh078

Teakle, G., and Gilmartin, P. 1998. Two forms of type IV zinc-finger motif and their 
610 kingdom-specific distribution between the flora, fauna and fungi. Trends Biochem. Sci.

611 23(3): 100-102. Available from https://doi.org/10.1016/S0968-0004(98)01174-8

612 Teakle, G.R., Manfield, I.W., Graham, J.F., and Gilmartin, P.M. 2002. Arabidopsis

613 thaliana GATA factors: organisation, expression and DNA-binding characteristics. Plant

614 Mol. Biol. 50(1): 43-56.

615 doi: $10.1023 / \mathrm{A}: 1016062325584$

616 Tsai, S.-F., Martin, D.I., Zon, L.I., D'Andrea, A.D., Wong, G.G., and Orkin, S.H. 1989.

617 Cloning of cDNA for the major DNA-binding protein of the erythroid lineage through

618 expression in mammalian cells. Nature 339(6224): 446-451. doi: $\underline{10.1038 / 339446 \mathrm{a} 0}$

619 Vanholme, B., Grunewald, W., Bateman, A., Kohchi, T., and Gheysen, G. 2007. The tify

620 family previously known as ZIM. Trends Plant Sci. 12(6): 239-244. Available from

621 https://doi.org/10.1016/j.tplants.2007.04.004

622 Wang, L., Yin, H., Qian, Q., Yang, J., Huang, C., Hu, X., and Luo, D. 2009. NECK

623 LEAF 1, a GATA type transcription factor, modulates organogenesis by regulating the

624 expression of multiple regulatory genes during reproductive development in rice. Cell

625 Res. 19(5): 598. doi: $\underline{10.1038 / \text { cr.2009.36 }}$

626 Wang, Y., Tang, H., DeBarry, J.D., Tan, X., Li, J., Wang, X., Lee, T.-h., Jin, H., Marler,

627 B., and Guo, H. 2012. MCScanX: a toolkit for detection and evolutionary analysis of

628 gene synteny and collinearity. Nucleic Acids Res. 40(7): e49-e49. Available from

629 https://doi.org/10.1093/nar/gkr1293

630 Wlodarski, M.W., Collin, M., and Horwitz, M.S. 2017. GATA2 deficiency and related 
myeloid neoplasms. Semin Hematol. 54:81-86

632 Available from https://doi.org/10.1053/j.seminhematol.2017.05.002

633 Wong, K.H., Hynes, M.J., and Davis, M.A. 2008. Recent advances in nitrogen regulation:

634 a comparison between Saccharomyces cerevisiae and filamentous fungi. Eukaryot. Cell 635 7(6): 917-925.

636 doi: $\underline{10.1128 / \text { EC.00076-08 }}$

637 Xu, F., Xi, Z.-m., Zhang, H., Zhang, C.-j., and Zhang, Z.-w. 2015. Brassinosteroids are

638 involved in controlling sugar unloading in Vitis vinifera 'Cabernet Sauvignon'berries

639 during véraison. Plant Physiol. Biochem. 94: 197-208. Available from

640 https://doi.org/10.1016/j.plaphy.2015.06.005

641 Yoo, S.-D., Cho, Y.-H., and Sheen, J. 2007. Arabidopsis mesophyll protoplasts: a

642 versatile cell system for transient gene expression analysis. Nature protocols 2(7): 1565.

643 doi: $10.1038 /$ nprot.2007.199

644 Zhang, X., Zhou, Y., Ding, L., Wu, Z., Liu, R., and Meyerowitz, E.M. 2013.

645 Transcription repressor HANABA TARANU controls flower development by integrating

646 the actions of multiple hormones, floral organ specification genes, and GATA3 family

647 genes in Arabidopsis. Plant Cell 25(1): 83-101. Available from

648 https://doi.org/10.1105/tpc.112.107854

649 Zhang, Y., Zhen, L., Tan, X., Li, L., and Wang, X. 2014. The involvement of hexokinase

650 in the coordinated regulation of glucose and gibberellin on cell wall invertase and sucrose

651 synthesis in grape berry. Mol. Biol. Rep. 41(12): 7899-7910. doi: $\underline{10.1007 / s 11033-014-}$ 
652

653

654

655

656

657

658

659

660

661

662

663

664

665

666

667

668

669

670

671

672

Zhao, F.-L., Li, Y.-J., Hu, Y., Gao, Y.-R., Zang, X.-W., Ding, Q., Wang, Y.-J., and Wen, Y.-Q. 2016. A highly efficient grapevine mesophyll protoplast system for transient gene expression and the study of disease resistance proteins. Plant Cell, Tissue Organ Cult 125(1): 43-57. doi: $\underline{10.1007 / \mathrm{s} 11240-015-0928-7}$

Zhao, Y., Medrano, L., Ohashi, K., Fletcher, J.C., Yu, H., Sakai, H., and Meyerowitz, E.M. 2004. HANABA TARANU is a GATA transcription factor that regulates shoot apical meristem and flower development in Arabidopsis. Plant Cell 16(10): 2586-260. Available from https://doi.org/10.1105/tpc.104.024869

\section{Figure legends}

Fig. 1 Phylogenetic analysis of GATA proteins. The amino acid sequences from grape and Arabidopsis were used to construct a tree in MEGA 5 using the neighbor-joining method with 1000 bootstrap replicates. The parameters used in the tree construction were poisson model, uniform rates and pairwise deletion. The four clades are marked with different colors.

Fig. 2 Chromosomal locations and gene duplication of grape GATA genes. The chromosomal units are in 100,000 bases. The chromosome number is shown inside the circle. The tandem duplication and WGD/segmental duplication genes are connected by a blue curve and red curves, respectively.

Fig. 3 Gene structures and motif composition of grape GATA factors. a. Schematic diagram of conserved motifs in VvGATA proteins. Each colored box represents a 
673 conserved motif in the protein, the black lines represent the non-conserved sequences. $b$.

674 Exon/intron structures of the $V v G A T A$ genes. Yellow boxes represent exons, spaces

675 between the boxes correspond to introns, and blue boxes represent untranslated region.

676 The size of genes can be estimated using the horizontal scale.

677 Fig. 4 Expression profiles of the $V v G A T A$ genes in grape organs ( $V$. vinifera $\mathrm{L}$. cv.

678 Cabernet Sauvignon). Expression was analyzed by real-time PCR using grape Actin1 as

679 reference gene. The gene expression level in different organs showing the highest CT

680 values was set to 1 . Data are presented in two separate graphs, $a$ and $b$; genes in b display

681 the highest relative expression levels. Data are presented as mean $\pm \mathrm{SD}$. There were two

682 biological replicates and the expression level of each was calculated from the mean value

683 of three technical replicates. c. Expression patterns of $V v G A T A$ genes in different berry

684 development stages from the RNA sequence; the berry samples of $V$. vinifera $\mathrm{L}$. cv.

685 Cabernet Sauvignon were collected at fruit set (F), veraison (V), and ripening (R). The

686 color scale represents transcript abundance which was the normalized FPKM values.

687 Green indicates low levels, black indicates a medium level and red indicates high levels

688 of transcript abundance.

689 Fig. 5 Subcellular localization of VvGATA proteins. The control 35S::GFP construct,

690 VvGATA14-GFP, VvGATA15-GFP, and VvGATA18-GFP fusion proteins were

691 transiently expressed in tobacco (N. benthamiana) protoplasts. The GFP green

692 fluorescence, chlorophyll autofluorescence, and bright-field images are shown (scale bar:

$69310 \mu \mathrm{m})$. 
694 Fig. 6 Expression patterns of subgroup III GATA genes in response to light and

695 phytohormones. a. Expression patterns of subgroup III genes under light/dark treatment.

696 b. IAA-induced expression patterns of subgroup III genes. c. GA3-induced expression

697 patterns of subgroup III genes. d. ABA-induced expression patterns of subgroup III

698 genes. e. EBR-induced expression patterns of subgroup III genes. The transcript levels

699 were analyzed by real-time PCR. Data are presented as mean \pm SD. There were two

700 biological replicates and the expression level of each was calculated from the mean value

701 of three technical replicates.

702

703

704 Table legends

705 Table 1 GATA genes in grape

706 Table 2 Common putative cis-elements in the promoter of the $V v G A T A$ genes

707

708

709

710

711

712

713

714 
726 Captions for Supplementary tables

727 Table S1 Primers used in this study

728 Table S2 GATA transcription factors in grape

729 Table S3 cis-elements in the promoter of the $V v G A T A$ genes 
Table 1 GATA genes in grape

\begin{tabular}{llllll}
\hline Gene name & GenBank & Gene ID & Genome location & Peptide & Sub \\
& accession no. & & & length & family \\
\hline VvGATA1 & XR_786007 & GSVIVT01025199001 & chr6:3427975..3432516 & 386 & I \\
VvGATA2 & XM_002283709 & GSVIVT01035614001 & chr4:2730001..2731754 & 196 & I \\
VvGATA3 & XM_002274836 & GSVIVT01024194001 & chr3:452476..454201 & 352 & I \\
VvGATA4 & XM_002277923 & GSVIVT01018180001 & chr15:13505485..13506854 & 255 & I \\
VvGATA5 & XM_002272726 & GSVIVT01018833001 & chr4:19467932..19469492 & 305 & I \\
VvGATA6 & XM_002282189 & GSVIVT01033385001 & chr8:21037307..21039075 & 265 & I \\
VvGATA7 & XM_002283992 & GSVIVT01017011001 & chr9:3439047..3440507 & 283 & I \\
VvGATA8 & XM_010651243 & GSVIVT01035048001 & chr5:1163455..1164868 & 153 & II \\
VvGATA9 & XM_010650837 & GSVIVT01018951001 & chr4:18421693..18423027 & 285 & II \\
VvGATA10 & XM_002279247 & GSVIVT01035406001 & chr4:1062805..1064236 & 306 & II \\
VvGATA11 & XM_002282137 & GSVIVT01015204001 & chr11:1817933..1819418 & 309 & II \\
VvGATA12 & XM_010662532 & GSVIVT01032362001 & chr14:26715231..26732295 & 367 & II \\
VvGATA13 & XM_002278333 & GSVIVT01031203001 & chr14:1203196..1204273 & 109 & II \\
VvGATA14 & XM_010657000 & GSVIVT01029593001 & chr9:20973735..20988914 & 244 & III \\
VvGATA15 & XM_002283717 & GSVIVT01009197001 & chr18:6040226..6058566 & 248 & III \\
VvGATA16 & XM_002270325 & GSVIVT01012518001 & chr3_random:451954..465083 & 254 & III \\
VvGATA17 & XM_010666122 & GSVIVT01009198001 & chr18:6060381..6094675 & 324 & III \\
VvGATA18 & XM_010646869 & GSVIVT01012523001 & chr3_random:527226..560073 & 188 & III \\
VvGATA19 & XM_019223388 & GSVIVT01013319001 & chr12:11878739..11880214 & 259 & IV \\
\hline
\end{tabular}


Table 2 Common putative cis-elements in the promoter of the $V v G A T A$ genes

\begin{tabular}{llll}
\hline cis-Element name & Sequence & Response & Number \\
\hline Box 4 & ATTAAT & Light & 16 \\
Box I & TTTCAAA & Light & 16 \\
I-box & GATANGG & Light & 12 \\
TCT-motif & TCTTAC & Light & 12 \\
G-box & CACGTG & Light & 14 \\
GA-motif & AAAGATGA & Light & 10 \\
GAG-motif & RGAGAKK & Light & 10 \\
Sp1 & CCRCCC & Light & 12 \\
Circadian & CAANNNNATC & Circadian & 13 \\
ABRE & CACGTG & Abscisic acid & 10 \\
TCA-element & TCATCTTTTT & Salicylic acid & 10 \\
MBS & YAACTG & Drought & 12 \\
TC-rich repeats & TTTTCTT & Defense and stress & 14 \\
HSE & AAAAAATTYS & Heat stress & 16 \\
ARE & TGGTTT & Anaerobic induction & 13 \\
Skn-1_motif & GTCAT & Endosperm & 16 \\
5 UTR Py-rich stretch & TTTCTTCTCT & Conferring high transcription levels & 11 \\
\hline
\end{tabular}

Number, a frequency of each cis-element appeared in $19 V v G A T A$ gene promoters. 


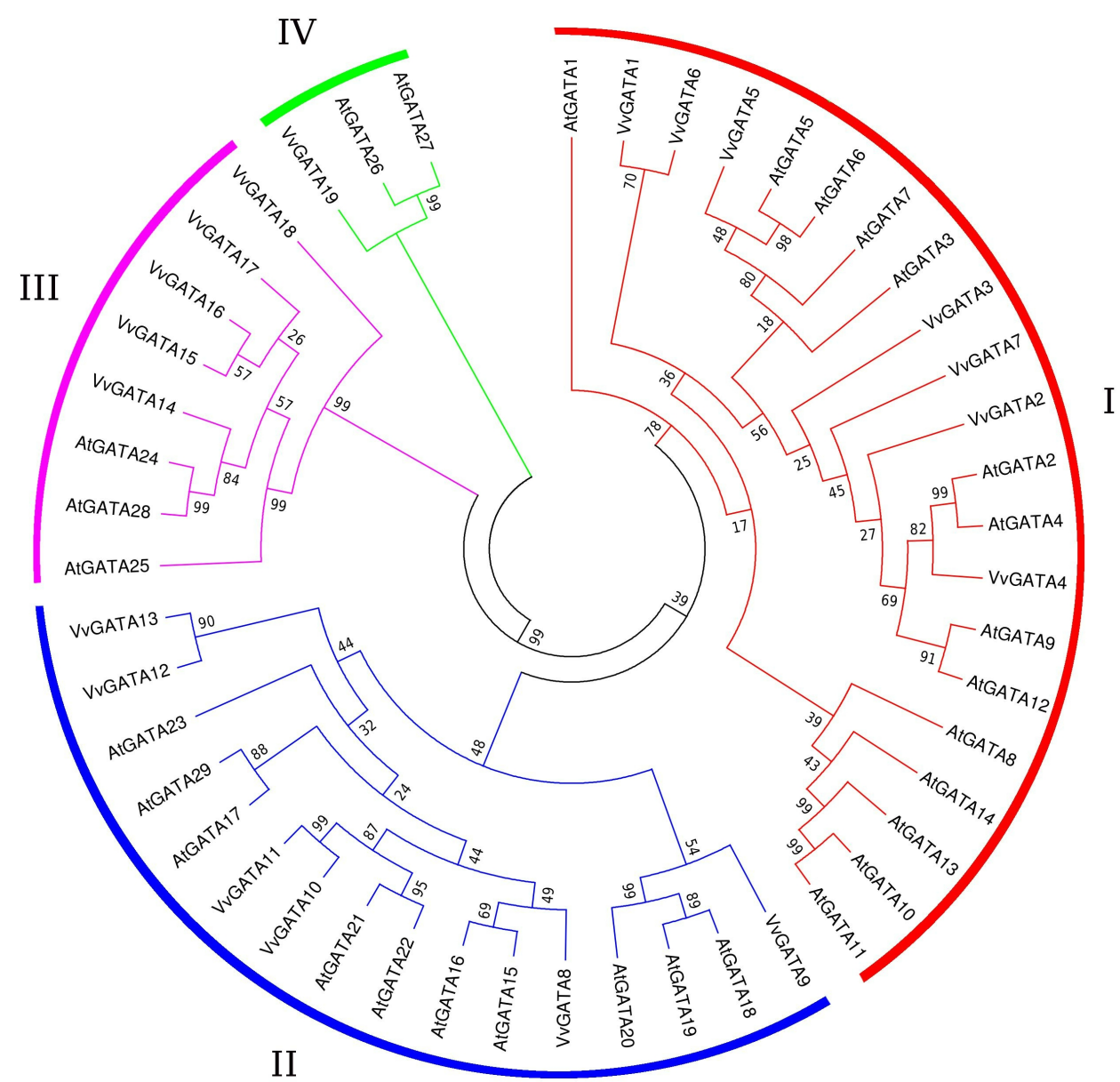

Figure 1 Phylogenetic analysis of GATA proteins.

$223 \times 223 \mathrm{~mm}(300 \times 300 \mathrm{DPI})$ 


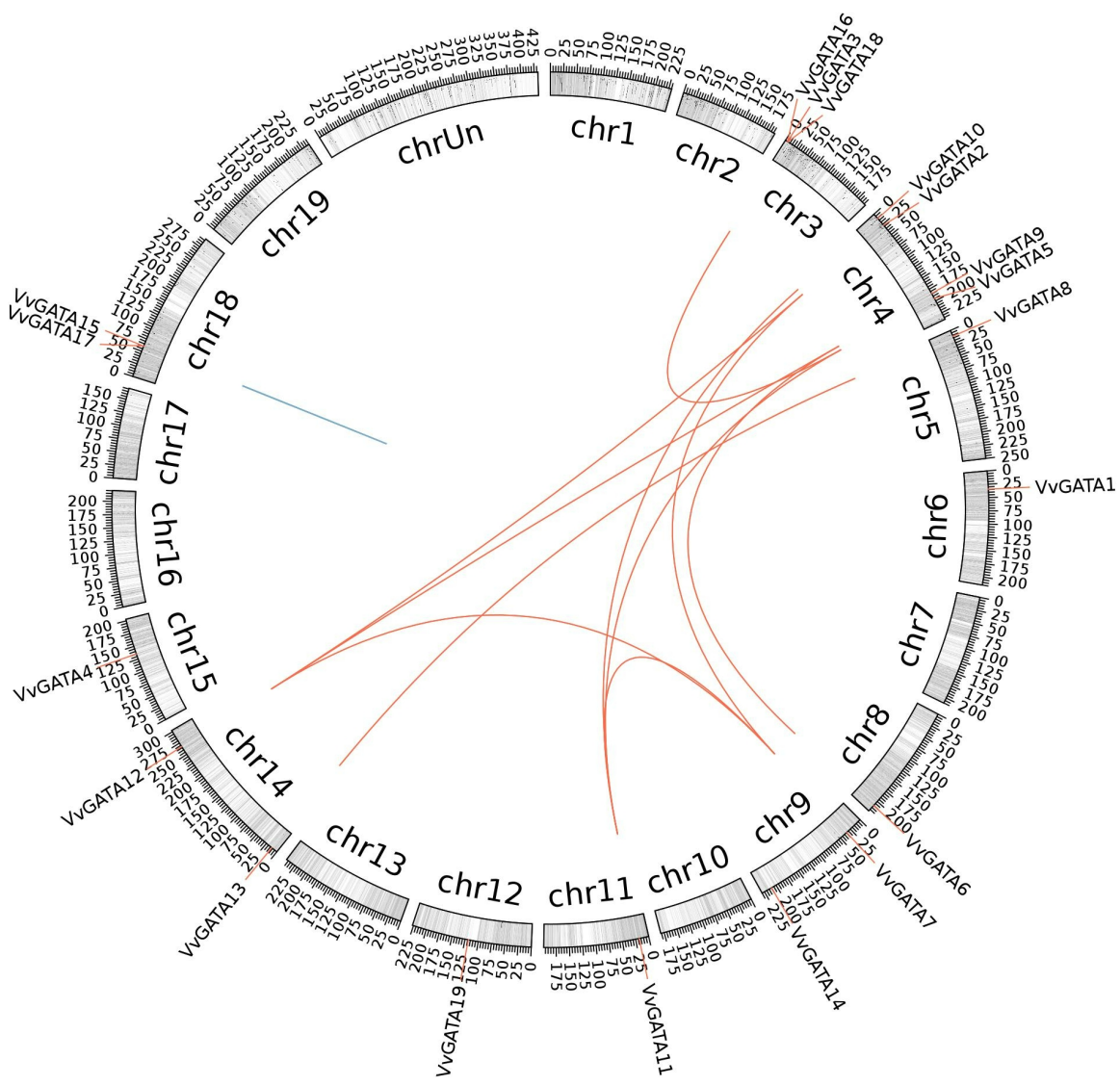

Figure 2 Chromosomal locations and gene duplication of grape GATA genes.

$181 \times 164 \mathrm{~mm}(300 \times 300 \mathrm{DPI})$ 

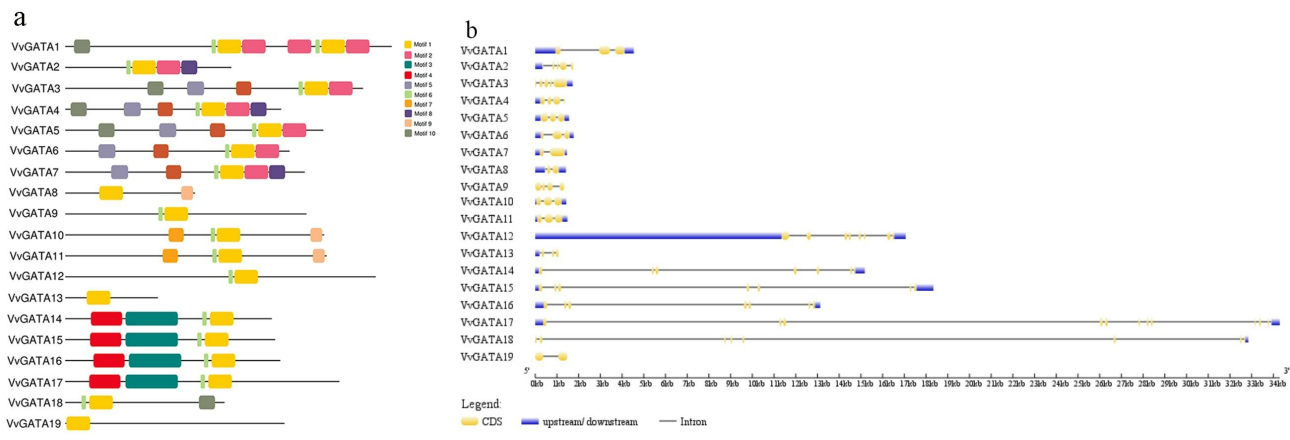

Figure 3 Gene structures and motif composition of grape GATA factors

$221 \times 73 \mathrm{~mm}(300 \times 300 \mathrm{DPI})$ 

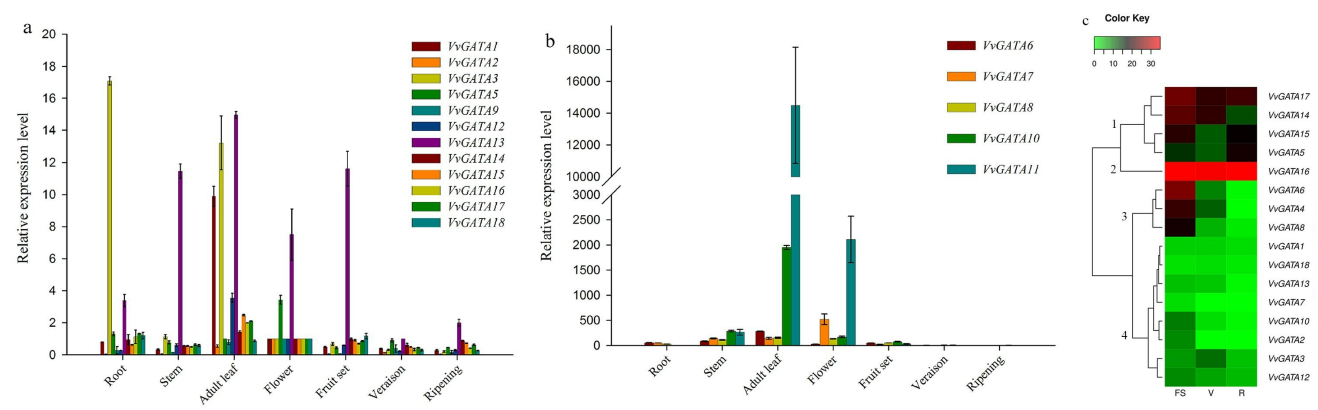

Figure 4 Expression profiles of the VvGATA genes in grape organs $254 \times 76 \mathrm{~mm}(300 \times 300 \mathrm{DPI})$ 


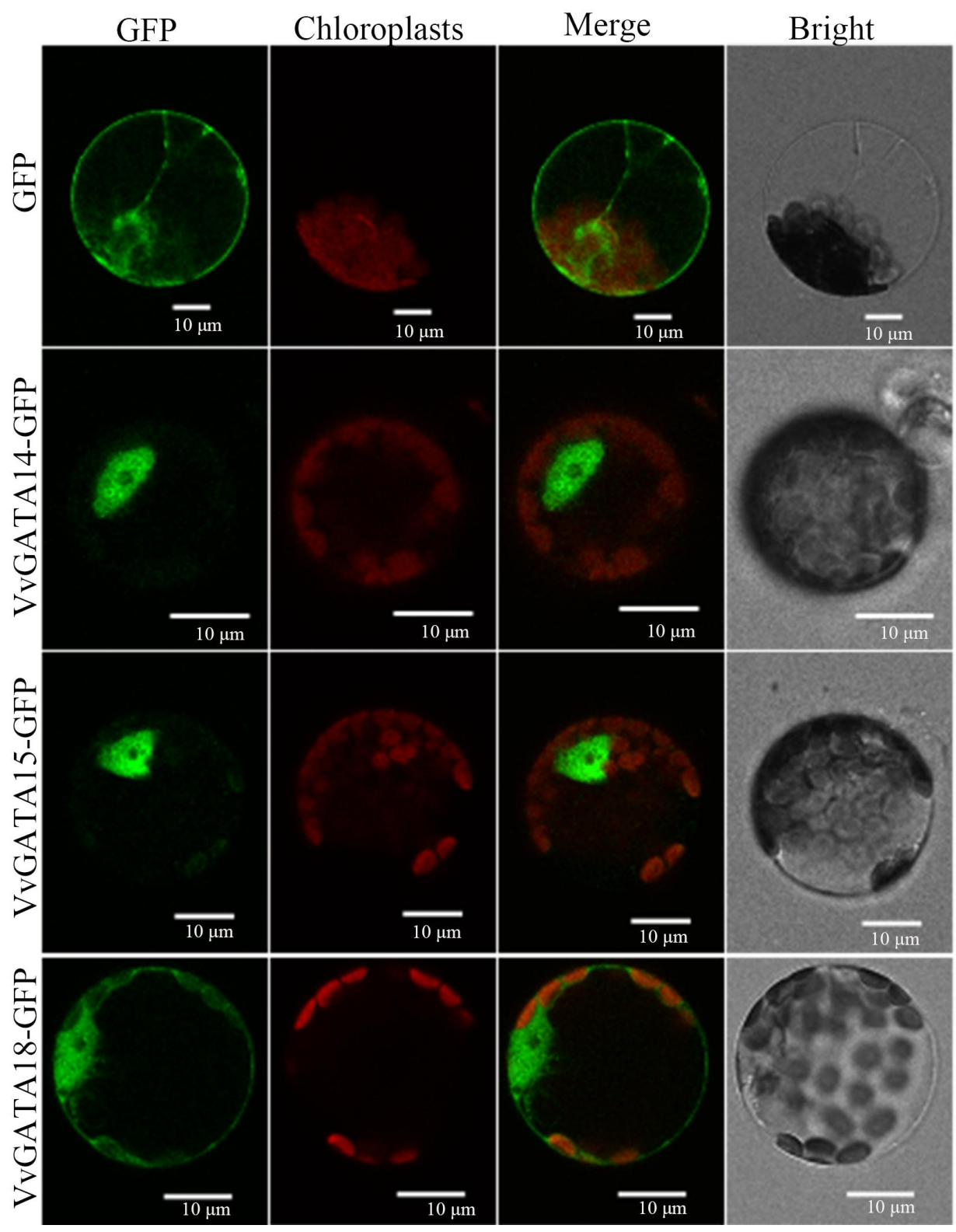

Figure 5 Subcellular localization of VvGATA proteins.

$142 \times 178 \mathrm{~mm}(300 \times 300 \mathrm{DPI})$ 

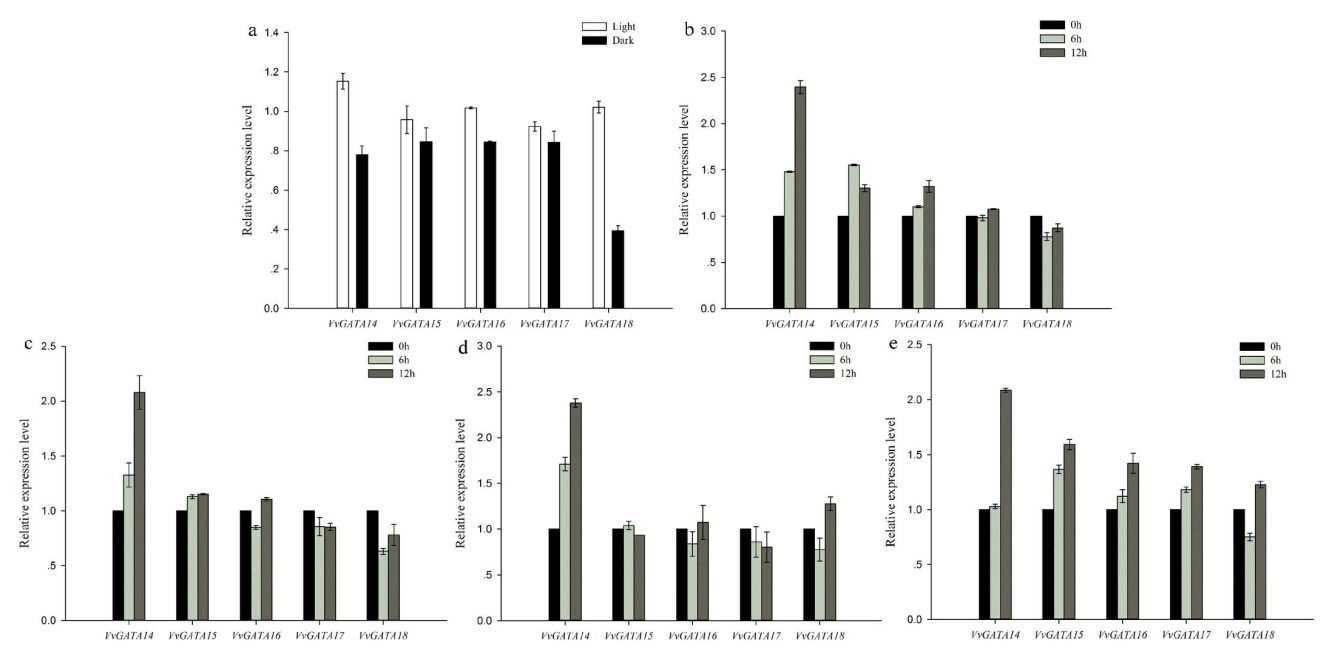

Figure 6 Expression patterns of subgroup III GATA genes in response to light and phytohormones

$181 \times 87 \mathrm{~mm}(300 \times 300 \mathrm{DPI})$ 